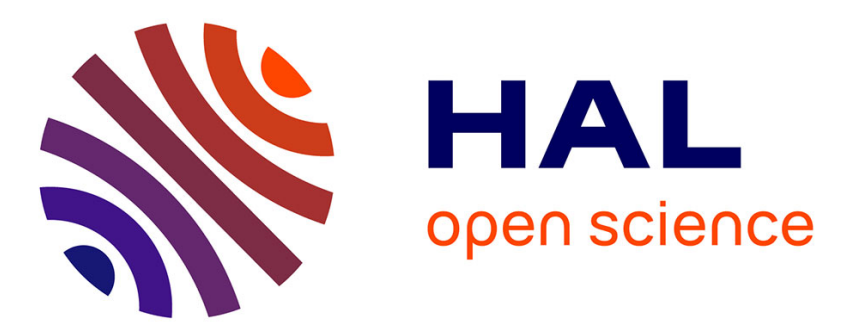

\title{
Optimal observer design for disturbed state affine systems
}

Francisco González de Cossío, Madiha Nadri, Pascal Dufour

\section{To cite this version:}

Francisco González de Cossío, Madiha Nadri, Pascal Dufour. Optimal observer design for disturbed state affine systems. 2018 Annual American Control Conference (ACC), Jun 2018, Milwaukee, United States. 10.23919/ACC.2018.8430762 . hal-01896047

\section{HAL Id: hal-01896047 https://hal.science/hal-01896047}

Submitted on 15 Oct 2018

HAL is a multi-disciplinary open access archive for the deposit and dissemination of scientific research documents, whether they are published or not. The documents may come from teaching and research institutions in France or abroad, or from public or private research centers.
L'archive ouverte pluridisciplinaire HAL, est destinée au dépôt et à la diffusion de documents scientifiques de niveau recherche, publiés ou non, émanant des établissements d'enseignement et de recherche français ou étrangers, des laboratoires publics ou privés. 


\title{
Optimal observer design for disturbed state affine systems
}

\author{
Francisco González de Cossío, Madiha Nadri and Pascal Dufour
}

\begin{abstract}
This paper investigates the problem of designing a robust high-gain observer for a class of disturbed state affine systems. The considered non-autonomous system takes into account both dynamic and output disturbances. The strategy is to provide a bound for the estimation error, independently of the initial condition, and to develop an algorithm for its minimization. The two arguments of this minimization problem are the tuning parameter of the observer and the systems input. This approach provides a new method to simultaneously design the input of the system and the tuning of the observer in order to minimize the effect of disturbances in state estimation.
\end{abstract}

\section{INTRODUCTION}

The problem of the design of asymptotic state observers for nonlinear systems is an important topic in control theory. It is often the case that the internal states of a given system are not measurable. The estimation of the unmeasured states of the system with an observer provides useful information to achieve different goals such as: feedback control [1], supervision and diagnostic, identification and, more recently, input design [2], [3]. On the other hand, accurate modeling of physical phenomena can be challenging and the measurement of the system output is often inexact. One way to face these challenges is by considering disturbances in both the dynamics of the system and its output.

One of the well-known standard methods of nonlinear observer design is the high-gain observer [4]. A tuning parameter is used in this structure to allow an arbitrary rate of convergence of the estimation error. This has a major drawback in practice where disturbances usually affect the system. Namely, the tuning parameter is highly sensitive to noise. This leads to a well-known trade-off problem between efficient estimation and noise amplification [5], [6].

A typical strategy to use in this trade-off problem is to formulate an adaptive gain. Simply put, the gain is high when the state needs to be reconstructed and then it drops down to prevent noise amplification. In this context we mention [7] and more recently [8], [9] where the high-gain parameter is dynamic. Another case of adaptive high-gain observers can be seen in [10]. Here the author bounded the limiting estimation error by the magnitude of measurement disturbance for a specific kind of systems.

The authors in [5] considered a switched high-gain observer which they applied to a class of uniformly observable nonlinear systems. They provided a bound for the estimation error that shows the trade-off arising from the tuning parameter and then proceeded to switch the gain according to this.

F. González de Cossío, M. Nadri and P. Dufour are with the Université de Lyon, Université Claude Bernard Lyon 1, CNRS UMR 5007 LAGEP, 43 Boulevard du 11 Novembre 1918, 69100 Villeurbanne, France madiha.nadri-wolf@univ-lyonl.fr
In [11] the authors addressed the problem for systems with high dimension. They developed an observer that restricts the gain power to 2 for general systems of dimension $n$ at the price of having a $2 n-2$ dimensional state vector. On the other hand, the authors in [12] developed a constant gain observer for systems with a triangular structure that avoids the high-gain.

However, the systems involved in most of these studies are uniformly observable and must be represented in their canonical observability form. Although this form characterizes the observability of systems [13], it is not always easy to compute the transformation that takes the system into this form.

The topic that we investigate in this paper is mainly motivated by the practical problem of identification of unknown parameters using an observer. A common strategy to solve this problem is to design an input for the system that optimizes the approximation of these parameters. Indeed, due to the trade-off between convergence speed and noise sensitivity, the selection of the input has a considerable impact on the performance of the observer [2], [14], [15], [16]. Designing inputs with special characteristics is not a trivial issue and, in practice, it is usually done in a heuristic way. However, some notable exceptions can be seen in [17], [18].

In this paper, the problem of observer design for state affine non-autonomous systems in presence of disturbances is considered. This class of systems is not necessarily in canonical form. Two types of bounded time-varying disturbances are distinguished: measurement disturbances affecting the output of the system and model uncertainties involved in the dynamics of the system. We investigate the robustness to noise of the observer and quantify a bound for the limiting estimation error. This bound depends on both: the highgain parameter and the systems input. We then redefined the bound for inputs with a proper excitation, the so-called regular persistence [19]. We present a novel approach of simultaneous off-line design of the high-gain parameter and the system input by minimizing this bound. Therefore, this method provides a new degree of freedom, in the problem of noise attenuation, by considering not only the high-gain parameter but also the input to the system.

The paper is organized as follows: the preliminaries in Section II provide necessary notation and some of the assumptions for stating our results. In this section we also study the basic properties of the observer used in this paper. The first part of Section III presents a bound for the estimation error of the systems state. In the second part of Section III, this bound is redefined in order to develop our optimization 
algorithm. Finally, a simple academic case is discussed in Section IV and some final remarks are given in Section V.

\section{PReliminaries}

\section{A. Notation}

The following standard notations are used in this paper:

- $\mathbb{R}^{+}$stands for the non-negative real numbers and $\mathbb{R}^{n}$ for the $n$-dimensional Euclidean space. We consider elements of $\mathbb{R}^{n}$ as column vectors. $\mathbb{R}^{n \times m}$ is the set of all matrices of size $n \times m$ with real coefficients.

- $I_{n \times n}$ is the $n$-dimensional identity matrix

- The transpose of the real matrix $M$ is $M^{\prime}$

- The maximum and minimum eigenvalues of a real symmetric matrix $N$ are denoted respectively by $\lambda_{\max }(N)$ and $\lambda_{\min }(N)$

- $\|\cdot\|$ denotes the Euclidean norm of a vector or the spectral norm of a matrix. Recall that the spectral norm of $M$ is given by:

$$
\|M\|^{2}=\lambda_{\max }\left(M^{\prime} M\right)
$$

\section{B. Systems dynamics and observer design}

The class of state affine nonlinear systems under consideration is described by the following equations:

$$
\left\{\begin{array}{l}
\dot{x}(t)=A(u(t)) x(t)+d_{1}(t) \\
y(t)=C x(t)+d_{2}(t)
\end{array}\right.
$$

where $t \geq 0, x: \mathbb{R}^{+} \rightarrow \mathbb{R}^{n}$ is the state, $A: \mathbb{R} \rightarrow \mathbb{R}^{n \times n}$ is a continuous matrix functional, $u: \mathbb{R}^{+} \rightarrow \mathbb{R}$ is a continuous input, $d_{1}: \mathbb{R}^{+} \rightarrow \mathbb{R}^{n}$ and $d_{2}: \mathbb{R}^{+} \rightarrow \mathbb{R}$ are bounded and continuous disturbances, $C \in \mathbb{R}^{1 \times n}$ and $y: \mathbb{R}^{+} \rightarrow \mathbb{R}$ is the output. We omit the dependence on time in the notation when no confusion might arise. [14]:

In this paper we consider the observer developed in [15],

$$
\left\{\begin{array}{l}
\dot{\widehat{x}}=A(u) \widehat{x}+S^{-1} C^{\prime}(y-C \widehat{x}) \\
\dot{S}=-\theta S-A(u)^{\prime} S-S A(u)+C^{\prime} C
\end{array}\right.
$$

where $\widehat{x}: \mathbb{R}^{+} \rightarrow \mathbb{R}^{n}$ is the state estimate, $S: \mathbb{R} \rightarrow \mathbb{R}^{n \times n}$ is the solution to the Riccati equation in (2) and $\theta$ is a positive tuning parameter. Notice that $S$ depends on both the input $u$ and the parameter $\theta$.

It is known that system (1) is not uniformly observable. In fact, this is closely related to the boundedness of the eigenvalues of $S(t)$. Is it not difficult to check that

$$
\begin{aligned}
& S(t)=\exp (-\theta t) \phi(0, t)^{\prime} S(0) \phi(0, t)+ \\
& \exp (-\theta t) \int_{0}^{t} \exp (\theta \tau) \phi(\tau, t)^{\prime} C^{\prime} C \phi(\tau, t) d \tau,
\end{aligned}
$$

where $\Phi$ stands for the transition matrix associated with $A$ and $u$. Namely, the unique solution on $\mathbb{R}^{+} \times \mathbb{R}^{+}$to

$$
\left\{\begin{array}{l}
\frac{\partial \phi(\tau, t)}{\partial \tau}=A(u(\tau)) \phi(\tau, t) \\
\phi(\tau, \tau)=I_{n \times n}
\end{array}\right.
$$

From the expression of $S(t)$ in (3) we can see that, if $S(0)$ is symmetric and positive definite, then $S(t)$ remains this way for all positive $t$.

Assumption 1: $S(0)$ is a symmetric and positive definite matrix in $\mathbb{R}^{n \times n}$.

Unfortunately, the eigenvalues of $S(t)$ can still be arbitrarily close to zero for the so-called singular inputs; inputs where observability is lost. We add the next assumption to avoid this situation.

Assumption 2: There exist real positive constants $c_{1}(u, \theta)$ and $c_{2}(u, \theta)$ such that if $t$ is large enough then we have

$$
\begin{aligned}
\lambda_{\max }\left(S^{2}(t)\right) & \leq c_{1}(u, \theta), \\
\lambda_{\min }(S(t)) & \geq c_{2}(u, \theta) .
\end{aligned}
$$

The explicit dependencies of $c_{i}$ on $u$ and $\theta$ are usually omitted to simplify the notation.

The authors in [15], [14] considered system (1) without disturbances, that is, with $d_{1}$ and $d_{2}$ set to zero. They show that, under a proper input excitation, the observer in (2) makes the estimation error

$$
e:=x-\widehat{x}
$$

converge to zero exponentially fast. Moreover, any desired rate of convergence may be achieved by increasing the tuning parameter $\theta$. Unfortunately, this is no longer true when disturbances are considered. Nevertheless, the estimation error of the states can be bounded. This bound is developed in the following section.

\section{ROBUSTNESS OPTIMIZATION BASED ON LYAPUNOV TECHNIQUES}

The estimation problem depends on finding a quantifiable bound for the estimation error $e$ in the case of dynamic and output disturbances. Naturally, this bound will depend on the magnitude of these disturbances. By minimizing the estimation error bound over an admissible space, we provide a systematic way to find an optimal excitation $u$ and an optimal observer tuning $\theta$ in terms of noise robustness.

\section{A. Bound on the estimation error}

One of the usual Gronwall-type lemmas is instrumental to the proof of our main result, see for example [20].

Lemma 1: Let $t_{0}<t_{1}$ be non-negative real numbers and $f, g:\left[t_{0}, t_{1}\right] \rightarrow \mathbb{R}$ continuous. Suppose also that $h:\left[t_{0}, t_{1}\right] \rightarrow \mathbb{R}$ is of class $C^{1}$ and that on their domain,

$$
\dot{h} \leq f h+g .
$$

Then, the next inequality holds for all $t$ in $\left[t_{0}, t_{1}\right]$ :

$h(t) \leq h\left(t_{0}\right) \exp \left(\int_{t_{0}}^{t} f(s) d s\right)+\int_{t_{0}}^{t} \exp \left(\int_{s}^{t} f(r) d r\right) g(s) d s$. 
For the sake of clarity, we denote the limiting disturbance magnitudes as

$$
\begin{aligned}
& L_{1}=\limsup _{t \rightarrow \infty}\left\|d_{1}(t)\right\|, \\
& L_{2}=\limsup _{t \rightarrow \infty}\left\|d_{2}(t)\right\| .
\end{aligned}
$$

Theorem 1: Consider systems (1) and (2). Suppose that Assumption 1 and Assumption 2 are both fulfilled. Based on the definitions given in (5), (6) and (7), the following inequality is satisfied:

$$
\limsup _{t \rightarrow \infty}\|e(t)\| \leq \frac{2 L_{1} c_{1}+2 L_{2} \sqrt{c_{1} \lambda_{\max }\left(C^{\prime} C\right)}}{c_{2}\left(\theta \sqrt{c_{1}}+\lambda_{\min }\left(C^{\prime} C\right)\right)} .
$$

Proof : We use the usual Lyapunov techniques. For this we define $V: \mathbb{R}^{n} \times \mathbb{R}^{+} \rightarrow \mathbb{R}^{+}$along the error as

$$
V(t, e(t)):=e(t)^{\prime} S(t) e(t) .
$$

By the chain rule, we have along the error trajectories that

$$
\begin{aligned}
\dot{V} & =2 e^{\prime} S \dot{e}+e^{\prime} \dot{S} e \\
& =2 e^{\prime} S\left(A(u) x+d_{1}-A(u) \hat{x}\right. \\
& \left.-S^{-1} C^{\prime}\left(C x+d_{2}-C \hat{x}\right)\right) \\
& +e^{\prime}\left(-\theta S-A(u)^{\prime} S-S A(u)+C^{\prime} C\right) e .
\end{aligned}
$$

If we rearrange the equality shown above,

$$
\begin{aligned}
\dot{V} & =2 e^{\prime} S A(u) e-2 e^{\prime} C^{\prime} C e-2 e^{\prime} C^{\prime} d_{2} \\
& +2 e^{\prime} S d_{1}-\theta e^{\prime} S e-e^{\prime} A(u)^{\prime} S e \\
& -e^{\prime} S A(u) e+e^{\prime} C^{\prime} C e \\
& =-e^{\prime} C^{\prime} C e-2 e^{\prime} C^{\prime} d_{2}+2 e^{\prime} S d_{1}-\theta e^{\prime} S e .
\end{aligned}
$$

This implies then that

$$
\begin{aligned}
\sqrt{V} & =\frac{1}{2 \sqrt{V}}\left(-e^{\prime} C^{\prime} C e-2 e^{\prime} C^{\prime} d_{2}+2 e^{\prime} S d_{1}\right. \\
& \left.-\theta e^{\prime} S e\right) .
\end{aligned}
$$

On the other hand, by the Cauchy-Schwartz inequality we have

$$
\begin{aligned}
e^{\prime} S d_{1} & \leq\|S e\|\left\|d_{1}\right\|, \\
-e^{\prime} C^{\prime} d_{2} & \leq\|C e\|\left\|d_{2}\right\| .
\end{aligned}
$$

Using (5) and denoting

$$
\begin{aligned}
\mu & =\frac{c_{1}}{c_{2}}, \\
\mu_{1} & =\frac{\lambda_{\max }\left(C^{\prime} C\right)}{c_{2}}
\end{aligned}
$$

we obtain:

$$
\begin{aligned}
& \|S e\|^{2}=e^{\prime} S^{2} e \leq \mu V, \\
& \|C e\|^{2}=e^{\prime} C^{\prime} C e \leq \mu_{1} V .
\end{aligned}
$$

Similarly,

$$
\frac{\lambda_{\min }\left(C^{\prime} C\right)}{\sqrt{c_{1}}} V(\cdot, e) \leq e^{\prime} C^{\prime} C e .
$$

Putting all this together, we get that

$$
\dot{\sqrt{V}} \leq \frac{\sqrt{V}}{2}\left(-\theta-\mu_{2}\right)+\sqrt{\mu}\left\|d_{1}\right\|+\sqrt{\mu_{1}}\left\|d_{2}\right\|,
$$

where

$$
\mu_{2}=\frac{\lambda_{\min }\left(C^{\prime} C\right)}{\sqrt{c_{1}}} .
$$

Note that $\mu$ is a positive constant and that $\mu_{1}$ and $\mu_{2}$ are non-negative constants. An inequality with this form is quite useful since Lemma 1 implies that for $t \geq t_{0}$,

$$
\begin{aligned}
& \sqrt{V(t, e(t))} \leq \\
& \exp \left(\frac{t}{4}\left(-\theta-\mu_{2}\right)\right) \sqrt{V\left(\frac{t}{2}, e\left(\frac{t}{2}\right)\right)}+ \\
& \sqrt{\mu} \int_{\frac{t}{2}}^{t} \exp \left(\left(-\theta-\mu_{2}\right) \frac{(t-s)}{2}\right)\left\|d_{1}(s)\right\| d s+ \\
& \sqrt{\mu_{1}} \int_{\frac{t}{2}}^{t} \exp \left(\left(-\theta-\mu_{2}\right) \frac{(t-s)}{2}\right)\left\|d_{2}(s)\right\| d s .
\end{aligned}
$$

Notice that for any non-zero constant $r$,

$$
\int_{\frac{t}{2}}^{t} \exp (r(t-s)) d s=-\frac{1}{r}+\frac{1}{r} \exp \left(r \frac{t}{2}\right)
$$

and since $V, d_{1}, d_{2}$ are bounded, we can take the superior limit on both sides of the inequality to conclude that

$$
\limsup _{t \rightarrow \infty} \sqrt{V(t, e(t))} \leq L_{1} \frac{2 \sqrt{\mu}}{\theta+\mu_{2}}+L_{2} \frac{2 \sqrt{\mu_{1}}}{\theta+\mu_{2}} .
$$

Since also

$$
\|e\|^{2} \leq \frac{1}{c_{2}} V
$$

the terms can be expanded and arranged to conclude the result.

\section{B. Optimization of the bound}

Theorem 1 provides a bound that can be minimized by playing simultaneously over positive tunings $\theta$ and admissible inputs $u$. To set this optimization problem correctly, we first need to specify a target function and its domain. We define the domain by considering inputs with the proper excitation, defined below, as in [15], [14]. We then redefine the bound in Theorem 1 as a function of $u$ and $\theta$ by specifying an explicit choice of $c_{1}(u, \theta)$ and $c_{2}(u, \theta)$ in (5).

Definition 1: The input $u$ is regularly persistent for system (1) with respect to the triplet of positive real numbers $\left(T, \alpha, t_{0}\right)$ if $t_{0} \geq T$ and if for all $t \geq t_{0}$ we have

$$
\int_{t-T}^{t} \Phi(\tau, t-T)^{\prime} C^{\prime} C \Phi(\tau, t-T) d \tau \geq \alpha I_{n \times n} .
$$

Here $\Phi$ stands for the transition matrix as defined in (4).

Bounds $c_{1}$ and $c_{2}$, as in (5), do exist for regularly persistent inputs. Moreover, these bounds have explicit 
expressions. We need the following assumption in order to formulate an explicit choice of $c_{1}$ and $c_{2}$.

Assumption 3: The matrix functional $A(u(t))$ is bounded. This means that

$$
\sigma=\sup _{t \geq 0}\|A(u(t))\|<\infty
$$

The next lemma is a direct consequence of the work developed in [19]. The proof relies in using the form of $S(t)$ given in (3) and basic properties of the transition matrix.

Lemma 2: Consider systems (1) and (2) and suppose Assumption 1 and Assumption 3 hold. For all $\theta>2 \sigma$ and $t \geq 0$ we have

$$
S(t) \leq \beta_{1} I_{n \times n}
$$

where

$$
\beta_{1}=\frac{\left\|C^{\prime} C\right\|}{\theta-2 \sigma}+\|S(0)\| .
$$

Moreover, if $u$ is also regularly persistent for system (1) with respect to $\left(T, \alpha, t_{0}\right)$ then for any $\theta>0$ and $t \geq t_{0}$ we have

$$
\beta_{2} I_{n \times n} \leq S(t)
$$

where

$$
\beta_{2}=\alpha \exp (-T(\theta+2 \sigma))
$$

Remark 1: Lemma 2 implies that Assumption 2 is satisfied when $u$ is regularly persistent. In fact, let us set the constants in (5) as:

$$
\begin{aligned}
& c_{1}=\beta_{1}^{2}, \\
& c_{2}=\beta_{2} .
\end{aligned}
$$

This selection of constants $c_{1}$ and $c_{2}$, together with Theorem 1, provide immediately the following corollary.

Corollary 1: Consider systems (1) and (2). Suppose that Assumption 1 and Assumption 3 are satisfied. If $u$ is regularly persistent for system (1) with respect to $\left(T, \alpha, t_{0}\right)$, then for any $\theta>2 \sigma$ :

$$
\begin{aligned}
& \limsup _{t \rightarrow \infty}\|e(t)\| \leq \\
& L_{1}\left(\frac{2(\theta-2 \sigma)\|S(0)\|+2\left\|C^{\prime} C\right\|}{\alpha \theta(\theta-2 \sigma) \exp (-T(\theta+2 \sigma))}\right)+ \\
& L_{2}\left(\frac{2 \sqrt{\left\|C^{\prime} C\right\|}}{\alpha \theta \exp (-T(\theta+2 \sigma))}\right) .
\end{aligned}
$$

The bound in Corollary 1 has an advantage over the bound in Theorem 1. Namely, the influence of the tuning parameter $\theta$ in the bound is now explicit. Our goal now is to define a functional that assigns to each pair $(u, \theta)$ the bound given by Corollary 1 . The optimal design of $u$ and $\theta$ is then given by the minimization of this functional.
Consider a set $\mathcal{U}$ of regularly persistent inputs for system (1) parametrized by a given bounded interval, say

$$
\mathcal{P}=\left[p_{\min }, p_{\max }\right] \text {. }
$$

Suppose also that

$$
\sigma^{*}=\sup _{u \in \mathcal{U}} \sigma(u)<\infty
$$

where $\sigma(u)$ is given in (10). This constraint on $\mathcal{U}$ is usually set in order to respect physical limitations of the system. In a similar way, the tuning parameter $\theta$ might have magnitude requirements and it needs also to verify the assumptions in Corollary 1 . Consider then the bounded interval

$$
\Theta=\left[\theta_{\min }, \theta_{\max }\right]
$$

for given

$$
\theta_{\max }>\theta_{\min }>2 \sigma^{*}
$$

In order to define a functional

$$
J: \Theta \times \mathcal{P} \rightarrow \mathbb{R}^{+}
$$

by using the bound in Corollary 1 , it suffices to assign to each $u \in \mathcal{U}$ a selection of the regularly persistent parameters $T$ and $\alpha$. We do this in a natural way. For each $u \in \mathcal{U}$ select and fix $T(u)$ such that the set

$$
\mathcal{R} \mathcal{P}(u)=\left\{\alpha \in(0, \infty) \mid \exists t_{0}>0 \text { satisfying (9) }\right\}
$$

is non-empty. Then simply set

$$
\alpha(u)=\sup \mathcal{R} \mathcal{P}(u) .
$$

Of course, the actual implementation of this design strategy of $u$ and $\theta$ is not trivial and needs to be adapted to the specific system under consideration. This strategy is based on the properties of the resulting functional $J$. For example, suppose the parametrization of $\mathcal{U}$ and the assignment $T(u)$ are done in a continuous way. Then $J$ results in a continuous functional with compact domain and a minimum is reached. There exist several tools available in the Matlab to perform the minimization. In particular, if $J$ is also strictly convex then the unique global minimum is easily obtained by the "fmincon" solver.

Let us summarize next the off-line design strategy of the optimal input $u^{*}$ and the optimal tuning parameter $\theta^{*}$.

1. Choose a regularly persistent input space $\mathcal{U}$,

2. Parametrize $\mathcal{U}$ continuously with $\mathcal{P}$ interval,

3. Choose a tuning parameter space $\Theta$,

4. Set a continuous length assignment $T(u)$ and compute $\alpha(u)$ as in (12),

5 . The optimal design is obtained by: $\left(\theta^{*}, p^{*}\right)=\underset{\theta \in \Theta, p \in \mathcal{P}}{\arg \min } J(\theta, p)$, where $J$ is given by the bound in Corollary 1 and where $u^{*} \in \mathcal{U}$ corresponds to $p^{*}$. 
The next section shows an academic example of this offline design strategy. The dimension of the considered state affine system is two and the input space $\mathcal{U}$ is set to be a family of parametrized cosine functions.

\section{ILLUSTRATION}

Here we apply the design strategy (13) to a specific academic case in order to illustrate the procedure. Let us consider system (1) in the specific case of $n=2$ and

$$
\begin{aligned}
A(u(t)) & =\left(\begin{array}{cc}
0 & u(t) \\
0 & 0
\end{array}\right), \\
C & =\left(\begin{array}{ll}
1 & 0
\end{array}\right) .
\end{aligned}
$$

The disturbances $d_{1}(t)$ and $d_{2}(t)$ will be discussed below. It is not needed to consider specific units for the time $t$.

Suppose that the physical limit of this system does not allow inputs with values outside the interval $[-1,1]$. For any input $u$, we obtain in this case that

$$
\sigma(u)=\sup _{t \geq 0}|u(t)|
$$

and we need to define $\mathcal{U}$ such that $\sigma^{*}=1$. On the other hand, the inputs have to be carefully selected since not every input to the system defined by (14) is regularly persistent [21]. Let us then choose the parametrized input space

$$
\mathcal{U}=\left\{u=\cos \left(p_{u} \cdot t\right) \mid p_{u} \in\left[p_{\min }, p_{\max }\right]\right\} .
$$

It is clear that in practice a realistic input frequency is lower and upper bounded. Since this an academic example, we instead use a rough approximation of the optimal frequency; Set $p_{\min }=0.1$ and $p_{\max }=14$. In a similar fashion, we set a top tuning of $\theta_{\max }=9$. Finally, following the hypotheses of Corollary 1 , we select $\theta_{\text {min }}=2.1>2 \sigma^{*}$.

We proceed to show that the inputs $u \in \mathcal{U}$ are regularly persistent with respect to their period

$$
T(u)=\frac{2 \pi}{p_{u}}
$$

and we give a formula for $\alpha(u)$. Since $A(u(t)) A(u(s))=0$ for any $t, s \geq 0$, the transition matrix is simply given by:

$$
\begin{aligned}
\Phi_{u}(s, t) & =\exp \int_{t}^{s} A(u(\tau)) d \tau \\
& =\left(\begin{array}{cc}
1 & \int_{t}^{s} u(\tau) d \tau \\
0 & 1
\end{array}\right),
\end{aligned}
$$

see for example [22]. It follows that the observability grammian in the left hand side of inequality (9) is the symmetric matrix $G(u, t)$ given by:

$$
\left(\begin{array}{cc}
T(u) & \int_{t-T(u)}^{t} \int_{t-T(u)}^{s} \cos \left(p_{u} \cdot \tau\right) d \tau d s \\
(\star) & \int_{t-T(u)}^{t}\left(\int_{t-T(u)}^{s} \cos \left(p_{u} \cdot \tau\right) d \tau\right)^{2} d s
\end{array}\right) .
$$

Then $u$ is regularly persistent if we can find $\alpha>0$ such that the matrix $G(u, t)-\alpha I$ has non-negative trace and nonnegative determinant for all $t \in\left[T, T+p_{u}\right]$. After some easy computations, this is equivalent to stating

$$
\begin{array}{r}
\frac{2 \pi}{p_{u}}+\frac{\pi}{p_{u}^{3}}-2 \alpha \geq 0, \\
\alpha^{2}-\left(\frac{2 \pi}{p_{u}}+\frac{3 \pi}{p_{u}^{3}}\right) \alpha+\frac{2 \pi^{2}}{p_{u}^{4}} \geq 0 .
\end{array}
$$

Finally, the largest admissible $\alpha(u)$ is the smallest root of the polynomial on the left hand side of the inequality in (15).

Let us now proceed with step five of the design strategy in (13). We suppose that the system is being affected by a twodimensional dynamic disturbance $d_{1}(t)$ represented as pairs of uniform random numbers between 0 and $\sqrt{200}$. Similarly, the output measurements are corrupted by uniformly random noise between 0 and 10 . Hence, $L_{1}=20$ and $L_{2}=10$. We fix $S(0)$ as the identity matrix and the functional $J$ is fully determined. It can be shown that the resulting functional $J$, specified by the bound in Corollary 1 , is in fact strictly convex and continuous in this case. We run the "fmincon" solver in Matlab to continue with step five in (13). The solver outputs immediately the optimal design:

$$
\left(\theta^{*}, p_{u}^{*}\right)=(3.3024,11.1651) \text {. }
$$

Consider system (1) and its observer (2) in the particular case of (14). Our goal now is to implement the optimal input and tuning given by (16) and to compare the performance of this optimal selection against other combinations of inputs and tunings. The measure of performance used here is given by the mean of the norm of the real estimation error over a fix time length. Several simulations of the system were run by setting an initial error derived from $x(0)^{\prime}=$ $(1,1)$ and $\hat{x}(0)^{\prime}=(0,0)$. Then the mean of the norm of the estimation error was computed over 50 time units for different combinations of tunings and inputs. The results can be seen in Table I. It is essential to notice that these values do not correspond to the functional $J$ but to the actual estimation error. Although the optimization domain did not include $\theta<2.1, \theta=0.1$ was also simulated for comparison. The design of the observer given by (13) provided a close to optimal performance with a value of 9.9. Notice the almost convex behavior of the table column-wise and row-wise.

Other systems states and their estimations can be seen in Figure 1 for the first ten time units and for different inputs and tunings. Here we initialized the systems at $x(0)^{\prime}=$ $(10,0)$ and $\hat{x}(0)^{\prime}=(5,50)$. Small values of the tuning $\theta$ fail to approximate fast and correctly both states simultaneously (Figure 1, first and second row from the top). But if $\theta$ is too large then the effect of the noise gets amplified (Figure 1, bottom row, second column). The optimal combination $\theta^{*}$ and $u^{*}$ is shown in the third row from the top of Figure 1.

\section{CONCLUSION}

In this paper we addressed the problem of robustness to measurement noise and model uncertainties of a high-gain observer for state affine non-autonomous systems.

As a first step, we provided an upper bound for the limiting estimation error in norm that depends on the magnitude of the disturbances. Then, we reformulated the bound of the 
TABLE I

MEAN NORM OF THE REAL ESTIMATION ERROR; THE VALUE 9.9 CORRESPONDING TO THE STRATEGY DESIGN DEVELOPED HERE IS IN THE LOWER \%10.2.

\begin{tabular}{c|ccccccc}
$\theta / p_{u}$ & 0.1 & 4 & 6 & 8 & $\mathbf{1 1 . 1}$ & 12 & 14 \\
\hline 0.01 & 269.3 & 157.7 & 169.7 & 177.9 & 186.5 & 187.9 & 190.2 \\
0.5 & 36.6 & 19.3 & 20.0 & 21.3 & 21.6 & 21.7 & 24.3 \\
1 & 20.8 & 11.0 & 11.1 & 11.7 & 11.6 & 11.4 & 12.9 \\
$\mathbf{3 . 3}$ & 22.1 & 9.6 & 9.9 & 9.8 & $\mathbf{9 . 9}$ & 10.1 & 10.9 \\
5 & 24.1 & 9.8 & 10.2 & 10.2 & 10.4 & 10.7 & 11.6 \\
7 & 26.9 & 10.5 & 10.7 & 10.5 & 11.0 & 11.4 & 12.4 \\
9 & 29.1 & 11.3 & 11.4 & 11.1 & 11.7 & 12.1 & 13.3 \\
\hline
\end{tabular}
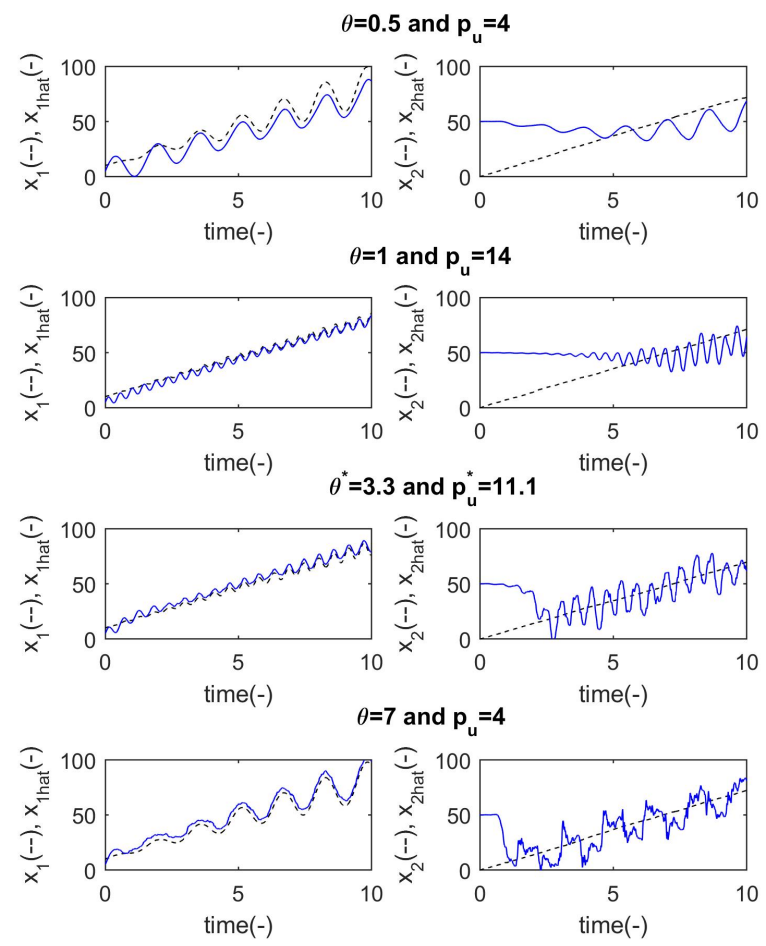

Fig. 1. Influence of $\theta$ and $p_{u}$ on the state estimation. In dotted line the states, in solid their estimations. The optimal design corresponds to the third row from the top.

estimation error for obtaining a more feasible optimization problem. Finally, using the obtained bound, we formulated an optimization algorithm for the off-line design of the input and the high-gain parameter. This approach provides a new degree of freedom to improve the performance of the observer with respect to measurement noise and unknown nonlinearities. Given the speed of the optimization algorithm, future studies will focus in the development of an on-line design of the observer. The work developed in this paper also opens a perspective for optimal input design for identification problems.

\section{REFERENCES}

[1] H. K. Khalil and L. Praly, "High-gain observers in nonlinear feedback control," International Journal of Robust and Nonlinear Control, vol. 24, no. 6, pp. 993-1015, Apr. 2014.

[2] P. Dufour, M. Nadri, and J. Qian, "An explicit optimal input design for first order systems identification," 17th IFAC Symposium on System Identification SYSID 2015, vol. 48, no. 28, pp. 344-349, Jan. 2015.

[3] J. Qian, M. Nadri, and P. Dufour, "Optimal input design for parameter estimation of nonlinear systems: case study of an unstable delta wing," International Journal of Control, vol. 90, no. 4, pp. 873-887, Apr. 2017.

[4] J.-P. Gauthier and I. Kupka, Deterministic observation theory and applications. Cambridge; New York: Cambridge University Press, 2001.

[5] J. Ahrens and H. Khalil, "High-gain observers in the presence of measurement noise: A switched-gain approach," Automatica, vol. 45, no. 4, pp. 936-943, Apr. 2009.

[6] L. K. Vasiljevic and H. K. Khalil, "Error bounds in differentiation of noisy signals by high-gain observers," Systems \& Control Letters, vol. 57 , no. 10 , pp. $856-862$, Oct. 2008.

[7] E. Bullinger and F. Allgower, "An adaptive high-gain observer for nonlinear systems," in Decision and Control, 1997. Proceedings of the 36th IEEE Conference on, vol. 5. IEEE, 1997, pp. 4348-4353.

[8] N. Boizot, E. Busvelle, and J.-P. Gauthier, "An adaptive high-gain observer for nonlinear systems," Automatica, vol. 46, no. 9, pp. 14831488, Sep. 2010

[9] R. G. Sanfelice and L. Praly, "On the performance of high-gain observers with gain adaptation under measurement noise," Automatica, vol. 47, no. 10, pp. 2165-2176, Oct. 2011.

[10] S. Battilotti, "Robust observer design under measurement noise with gain adaptation and saturated estimates," Automatica, vol. 81, pp. 7586, Jul. 2017.

[11] L. Wang, D. Astolfi, L. Marconi, and H. Su, "High-gain observers with limited gain power for systems with observability canonical form," Automatica, vol. 75, pp. 16-23, Jan. 2017.

[12] A. Zemouche, M. Boutayeb, and G. I. Bara, "Observers for a class of Lipschitz systems with extension to performance analysis," Systems \& Control Letters, vol. 57, no. 1, pp. 18-27, Jan. 2008.

[13] J.-P. Gauthier and G. Bornard, "Observability for any u (t) of a class of nonlinear systems," IEEE Transactions on Automatic Control, vol. 26 , no. 4, pp. 922-926, 1981.

[14] N. Couenne, "Synthèses d'observateurs de systèmes affines en l'état," Doctorate thesis, National Polytechnical Institute, 1988.

[15] G. Bornard, N. Couenne, and F. Celle, "Regular persistent observers for bilinear systems," Proceeding of the 29 international conference on nonlinear systems. New trend in nonlinear system theory. Vol 122 Springer Verlag, Jun. 1988.

[16] G. Besançon, "A Link between Output Time Derivatives and Persistent Excitation for Nonlinear Observers," 10th IFAC Symposium on Nonlinear Control Systems NOLCOS 2016, vol. 49, no. 18, pp. 493-498, Jan. 2016.

[17] I. Rubio Scola, G. Besançon, and D. Georges, "Input optimization for Observability of State Affine Systems," IFAC Proceedings Volumes, vol. 46, no. 2, pp. 737-742, 2013.

[18] G. Besançon, I. Rubio Scola, and D. Georges, "Input selection in observer design for non-uniformly observable systems," IFAC Proceedings Volumes, vol. 46, no. 23, pp. 664-669, 2013.

[19] G. Besançon, G. Bornard, and H. Hammouri, "Observer Synthesis for a Class of Nonlinear Control Systems," European Journal of Control, vol. 2, no. 3, pp. 176-192, 1996

[20] C. M. Hack1, Non-identifier Based Adaptive Control in Mechatronics: Theory and Application, ser. Lecture Notes in Control and Information Sciences. Springer International Publishing, 2017.

[21] G. Besançon, Ed., Nonlinear observers and applications, ser. Lecture notes in control and information sciences. Berlin ; New York: Springer, 2007, no. 363.

[22] H. Logemann and E. P. Ryan, Ordinary Differential Equations, ser Springer Undergraduate Mathematics Series. London: Springer London, 2014. 